\title{
Análisis exergético de un ciclo Brayton supercrítico con dióxido de carbono como fluido de trabajo
}

\section{Exergetic analysis of a supercritical Brayton cycle with carbon dioxide as working fluid}

DOI: http://dx.doi.org/10.17981/ingecuc.14.1.2018.15

Artículo de investigación científica. Fecha de recepción: 24/04/2018. Fecha de aceptación:21/06/2018.

\author{
Moisés Herrera Palomino \\ Universidad del Atlántico. Barranquilla (Colombia) \\ moisesherrera@mail.uniatlantico.edu.co \\ Edgardo Castro Pacheco \\ Universidad del Atlántico. Barranquilla (Colombia) \\ ercastro@mail.uniatlantico.edu.co \\ Jorge Duarte Forero \\ Universidad del Atlántico. Barranquilla (Colombia) \\ jorgeduarte@mail.uniatlantico.edu.co \\ Armando Fontalvo Lascano \\ Universidad de la Costa. Barranquilla (Colombia) \\ afontalv17@cuc.edu.co \\ Ricardo Vásquez Padilla \\ Southern Cross University. Lismore (Australia) \\ ricardo.vasquez.padilla@scu.edu.au
}

Para citar este artículo:

M. Herrera Palomino, E. Castro Pacheco, J. Duarte Forero, A. Fontalvo Lascano y R. Vásquez Padilla, "Análisis exergético de un ciclo Brayton supercrítico con dióxido de carbono como fluido de trabajo," INGE CUC, vol. 14, no. 1, pp. 159-170, 2018. DOI: https://doi. org/10.17981/ingecuc.14.1.2018.15

\section{Resumen}

Introducción- Actualmente, el modelado termodinámico de los ciclos de potencia es una herramienta muy atractiva con la cual se logra analizar y determinar cuán eficiente podría llegar a ser la combinación de distintos ciclos y/o la implementación de diversos componentes, que con sus diversas características y comportamientos impactan de forma positiva sobre la generación de energía. Además de ir ganando importancia en la utilización de tecnologías medioambientalmente amigables.

Objetivo- En este estudio se busca determinar el impacto de los parámetros de funcionamiento de un ciclo Brayton supercrítico con respecto a su comportamiento energético y exergético a medida que se realiza la variación de la temperatura del ciclo y demás condiciones de trabajo, tales como el uso de calentador y recalentador.

Metodología- Se realizó un modelo termodinámico para el análisis energético y exergético de 4 configuraciones de un ciclo Brayton supercrítico con dióxido de carbono como fluido de trabajo a variados niveles de temperatura y garantizando una presión máxima de $25 \mathrm{MPa}$.

Resultados- Los resultados obtenidos del modelo desarrollado y validado permitieron verificar que para las configuraciones con recalentamiento se presentan pérdidas totales de exergía consistentemente más bajas que para las configuraciones sin este. En conjunto, la temperatura de entrada a la turbina y las relaciones de presión tienen una influencia significativa sobre estas pérdidas, obteniéndose su valor mínimo a temperaturas de entre $800-850^{\circ} \mathrm{C}$.

Conclusiones- Las pérdidas totales de exergía son menores en las configuraciones que implementan el uso de recalentador que las que no lo usan. Se aprecia que con el uso de recalentador las pérdidas de exergía disminuyen en al menos un $3 \%$ a medida que aumenta la temperatura para todas las configuraciones.

Palabras clave-Modelamiento, energía, exergía, recalentamiento, ciclo Brayton

\begin{abstract}
Introduction- Nowadays, the thermodynamic modeling of the power cycles is conceived as an appropriate device which allows analyzing and determining the adaptability of several cycles as well the implementation and combination of a number of components whose characteristics and performing work appropriately on the generation of energy, beside of this, the relevant use of environmentally friendly technologies was taking into account as a relevant factor.

Objective- This research works intends to determine the impact of the performance parameters from the supercritical Brayton cycle related to its energetic and exergetic performance as the variation of the temperature of the cycle as well alternative working conditions are executed by using the reheater and heater systems.

Methodology- This research project used a thermodynamic model to carry out the energy and exergy analysis from four configurations of the supercritical Brayton cycle along carbon dioxide as a working fluid through several levels of temperature, also a maximum pressure of twenty five MPa was ratified. Results-The obtained results have shown the developed and assessed model allowed to demonstrate the configurations through reheat; as for energy there exist a consistently lack of it with regards to the use of the mentioned systems as these have been not configured. In addition the temperature related to the inlet of the turbine and the pressure ratios have a relevant influence on these lacks by obtaining its minimum value at temperatures between $800-850^{\circ} \mathrm{C}$.

Conclusions- It can be said that the total lack of exergy is minor as the configurations from the reheater system as it is applied, in other words it is estimated that through the use of the mentioned system the percentage of reduction aims to three percent as the temperatures increase for the whole configurations.

Keywords-Modeling, Energy, Exergy, Reheat, Brayton Cycle.
\end{abstract}




\section{INTRODUCCIÓN}

En la actualidad, la creciente demanda energética por la que se está atravesando origina la necesidad de desarrollar e implementar mejoras a los sistemas de generación de energía usados y, de esta forma, intentar encontrar una manera de lograr satisfacer tales exigencias energéticas. Son diversas las opciones que se han propuesto para suplir los crecientes índices de consumo de energía, como el análisis económico de fuentes energéticas renovables y no renovables desarrollado por [1]. Sin embargo, hoy por hoy, la selección y desarrollo de las tecnologías de generación de energía no van encaminadas solo a satisfacer la demanda generada por la población, sino a que esas tecnologías que lleguen a ser implementadas cumplan con los requerimientos medioambientales estipulados en la actualidad y logren mitigar cualquier tipo de impacto ecológico que resulte negativo debido a su aplicación.

El desarrollo de nuevas tecnologías de generación de energía es un tema de gran importancia a nivel mundial, sin embargo, otro ideal al que se le está dando mucha trascendencia y al que se están encaminando muchas de las recientes investigaciones es a la mejora de los sistemas actuales, de tal forma que se logre alcanzar un óptimo nivel de funcionamiento. Estos sistemas incluyen desde los más simples, como el sistema cilindro embolo [2], hasta los más complejos, como los relacionados con los ciclos de generación de potencia que maximicen la producción de energía sin afectar el medio ambiente. Entre las tecnologías a las que se les está apuntando la mirada con gran interés para lograr mejoras en su funcionamiento están las comprendidas por los ciclos térmicos de generación de potencia, entre los cuales, para alcanzar los niveles deseados de mejora, se está apostando a procedimientos que van desde modificaciones a los componentes que constituyen los ciclos, hasta la implementación y acople de éstas con otras tecnologías de generación. Como ejemplo de esto, se tiene el ciclo Rankine orgánico (ORC, por sus siglas en inglés), el cual es una variación del ciclo Rankine de vapor, y que hoy en día es el ciclo con mayor presencia en el mercado para potencias superiores a $200 \mathrm{~kW}$. Este ciclo no es más que un ciclo Rankine que usa fluidos orgánicos con bajo punto de ebullición como fluido de trabajo en lugar de vapor, esto con el fin de recuperar el calor de una fuente de baja temperatura [3].

Entre otros ciclos de potencia expuestos a constantes mejoras, se tienen el ciclo Kalina [4], el cual es una variación directa del ciclo Rankine en el que se utiliza una solución de dos fluidos con diferentes puntos de ebullición como fluido de trabajo. El ciclo Goswami [5], el cual es un nuevo ciclo termodinámico que cuenta con la combinación de un ciclo Rankine y un ciclo de generación por adsorción. Este ciclo ha sido estudiado mediante la implementación de diversas mezclas como fluido de trabajo, tales como la mezcla de agua con amonio [6], con la que se buscaba mejorar el comportamiento del ciclo bajo ciertas condiciones de operación. Se tiene también, el ciclo Antanoupulus [7], el cual es una variación del ciclo Kalina, en el cual se cambian las etapas de destilación-condensación del ciclo por un proceso de adsorción por destilación. Por otra parte, también se tiene el ciclo Brayton supercrítico, que ha adquirido un gran atractivo por el uso de dióxido de carbono como fluido de trabajo. Este ciclo fue propuesto inicialmente con el fin de recuperar calor de fuentes de baja temperatura mediante el uso de $\mathrm{CO}_{2}$ en estado supercrítico como fluido de trabajo. El dióxido de carbono se hace interesante para este ciclo de potencia debido a que muestra ciertas ventajas con su uso, tales como:

- No presenta un alto índice de toxicidad ni inflamabilidad

- Tiene bajos costos de capital en comparación con los fluidos de trabajo de otros ciclos

- Su relativo bajo nivel de temperatura y presión $\left(32{ }^{\circ} \mathrm{C}\right.$ y $\left.7.4 \mathrm{kPa}\right)$ para llegar al punto crítico en comparación con otros fluidos como el agua (374 ${ }^{\circ} \mathrm{C}$ y $22 \mathrm{MPa}$ )

Este último conlleva al diseño de componentes más compactos, de menor tamaño y menos complejos [8].

Desde un punto de vista medioambiental, la aplicación del ciclo Brayton supercrítico con dióxido de carbono $\left(\mathrm{s}-\mathrm{CO}_{2}\right)$ permite el aprovechamiento y explotación de recursos no convencionales, tales como la radiación solar [6] [9] [10], el calor residual de procesos industriales [11] [12], entre otras, lo que lo hace un ciclo responsable y amigable con el medio ambiente.

Son numerosos los estudios e investigaciones que se han centrado en analizar el comportamiento del ciclo Brayton s- $\mathrm{CO}_{2}$ con el fin de encontrar mejoras en su funcionamiento, y con esto, una mayor gama de aplicaciones, tal como lo propone [13] para aplicaciones nucleares. Por otra parte, [14] y [15] llevaron a cabo un estudio con diferentes configuraciones del ciclo Brayton, encontrando que las configuraciones del ciclo Brayton s- $\mathrm{CO}_{2}$ con refrigeración y recompresión parcial tienen mayor eficiencia térmica en comparación con un ciclo Brayton s- $\mathrm{CO}_{2}$ sencillo. Del mismo modo, [16] llevó a cabo el análisis de energía y exergía de un ciclo Brayton s- $\mathrm{CO}_{2}$ con recompresión, integrado con un reactor nuclear, y realizó un análisis de sensibilidad para investigar el efecto de los parámetros del ciclo en la eficiencia exergética. En [16] se encontró que las irreversibilidades de los intercambiadores de calor son más altas que las obtenidas en el compresor y la turbina. En [9] se analiza el comportamiento de un ciclo Brayton s$\mathrm{CO}_{2}$, integrado con una central de colectores solares, hallando que la eficiencia térmica del ciclo aumenta uniformemente con la temperatura. Además, en función de un análisis exergético, el colector solar 
presenta la más alta destrucción exergética mientras que componentes como turbinas y compresores tienen una mínima contribución a esta.

En este estudio, se presenta un análisis desde el punto de vista de la primera y segunda ley de la termodinámica para cuatro configuraciones de ciclo Brayton s- $\mathrm{CO}_{2}$ : ciclo Brayton simple, ciclo Brayton de recompresión (RC), ciclo Brayton de enfriamiento parcial (PC) y ciclo Brayton de compresión principal con interenfriamiento parcial (MC). El estudio realizado a las cuatro configuraciones mencionadas anteriormente cuenta con el modelado de estas tanto con el uso de recalentamiento como sin el uso de este. Las condiciones óptimas de operación del ciclo Brayton s- $\mathrm{CO}_{2}$ son determinadas a partir de una previa optimización de los parámetros de funcionamiento, tales como las relaciones de presión y el Split Ratio (SR), que no es más que la fracción del flujo másico que entra a la línea caliente del recuperador de baja temperatura en todas las configuraciones a excepción de la configuración simple hasta lograr las máximas eficiencias a las condiciones de operación pre-establecidas. El ciclo en cuestión fue analizado sin tener en cuenta los efectos causados por la aplicación de una fuente térmica en particular, tal como se aprecia en la Fig. 1. De esta forma, la fuente térmica es tomada como una "caja negra", de la cual solo se extrae el calor requerido para el funcionamiento del ciclo sin tener en cuenta el aporte exergético que esta pueda causar.

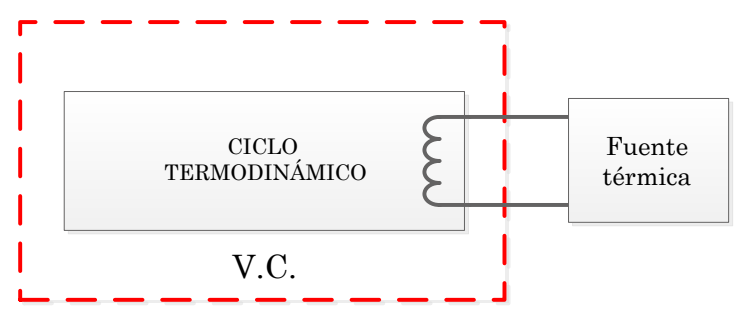

Fig. 1. Esquema representativo de la delimitación del volumen de control de las configuraciones de ciclo Brayton s- $\mathrm{CO}_{2}$ a analizar. Fuente: elaboración propia.

\section{Configuraciones del Ciclo Brayton S-CO}

El componente o dispositivo que provee la energía de entrada necesaria para poner en funcionamiento el ciclo es tomado como una "caja negra" en la cual no son tenidos en cuenta sus efectos sobre el comportamiento de las configuraciones. Estas configuraciones están basadas en las modificaciones propuestas por los autores estudiados dentro de la revisión bibliográfica [9] [17]. La descripción general de las configuraciones analizadas en la Fig. 2 se presenta más adelante. En la Fig. 3 se presentan los diagramas de Temperatura-Entropía (T-S) para las cuatro configuraciones de ciclo Brayton s- $\mathrm{CO}_{2}$ a una temperatura de entrada de la turbina primaria de $700{ }^{\circ} \mathrm{C}$.

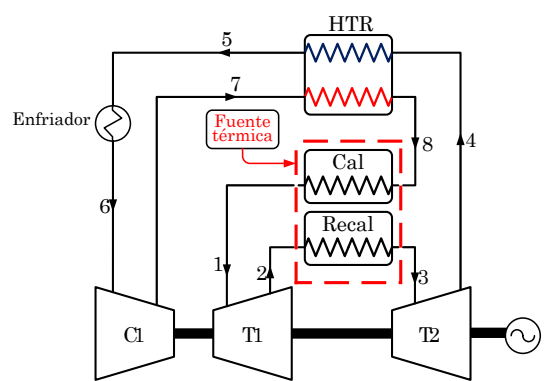

a)

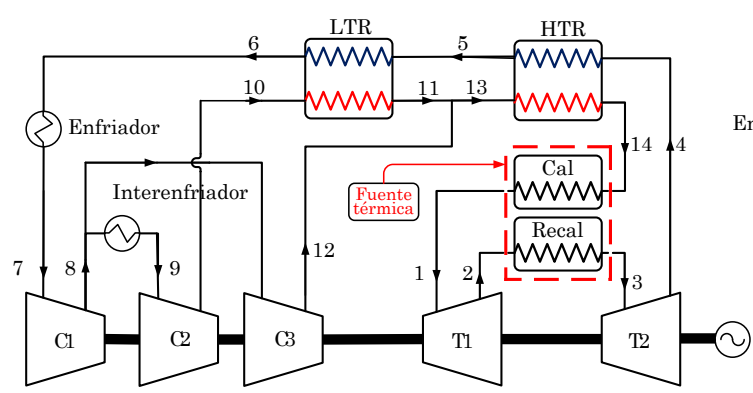

c)

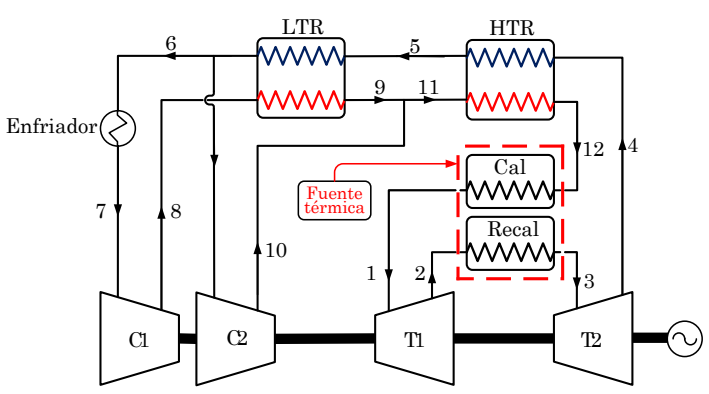

b)

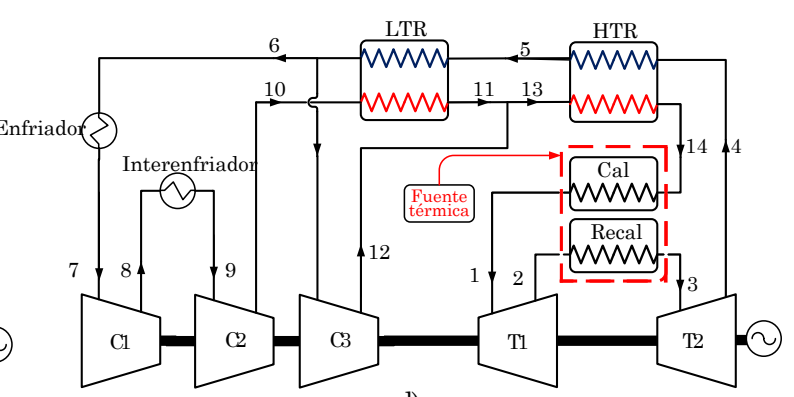

d)

Fig. 2. Configuraciones de ciclo Brayton $\mathrm{S}-\mathrm{CO}_{2}$. (a) Configuración simple con recalentamiento, (b) configuración $\mathrm{RC}$ con recalentamiento, (c) configuración PC con recalentamiento, (d) configuración MC con recalentamiento. Fuente: elaboración propia. 
Configuración simple (simple). La configuración simple se basa en un ciclo Brayton básico que incorpora un recuperador, como se puede observar en la Fig. 2-a. En este ciclo, el dióxido de carbono a alta temperatura y presión se expande a una presión intermedia a través de una turbina primaria (turbina 1) para luego ser recalentada y expandida a menor presión (turbina 2). Se usa un recuperador para aprovechar la energía de la corriente de salida de la turbina secundaria y precalentar la corriente que se dirige al calentador. Para aumentar la presión de la corriente de menor nivel energético que va al recuperador térmico, la corriente es previamente enfriada para luego ser comprimida en el compresor principal (compresor 1). Se aprecia que parte del trabajo producido por las turbinas se utiliza para accionar el compresor.

Configuración de recompresión (RC). Debido a la rápida variación de las propiedades termo-físicas del $\mathrm{CO}_{2}$ cerca de las condiciones críticas [18], la configuración del ciclo simple está limitada por problemas de temperatura de pinch-point (punto en el que se da la diferencia mínima de temperatura entre las curvas de frío y caliente de un proceso térmico) en el recuperador debido a la diferencia en la capacidad de transferencia de calor entre el lado caliente y el frío. Por lo tanto, se propone una configuración de ciclo Brayton $\mathrm{s}-\mathrm{CO}_{2}$ con recompresión, como se muestra en la Fig. 2b. Esta configuración es similar

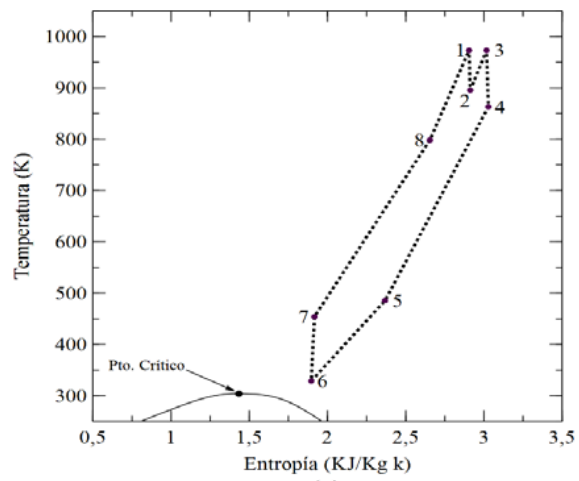

(a)

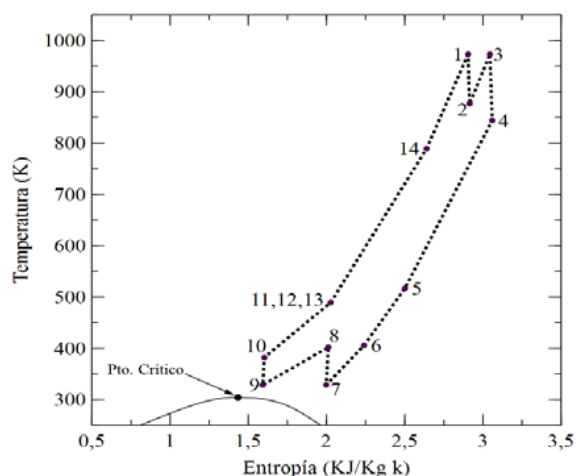

(c) a la configuración simple (la cual fue mencionada en el apartado anterior), con la diferencia de que se utilizan dos recuperadores (recuperador térmico de alta y recuperador térmico de baja) y un segundo compresor después de ambos regeneradores. Al igual que en la anterior configuración, parte del trabajo producido por las turbinas es también utilizado para accionar ambos compresores.

Configuración de enfriamiento parcial (PC). Ya que en las configuraciones anteriores parte del trabajo desarrollado por las turbinas es utilizado para accionar los compresores, se intenta reducir el trabajo consumido durante este proceso de compresión adicionando una etapa de enfriamiento entre las diferentes etapas de compresión [19], tal como se muestra en la Fig. 2c. El funcionamiento de esta configuración es similar a la presentada para el ciclo de recompresión descrito anteriormente.

Configuración de compresión principal con interenfriamiento parcial (MC). Como se puede apreciar en la Fig. 2d, en la configuración de enfriamiento parcial se hace uso de un compresor adicional (con respecto a los ciclos anteriormente explicados) y del uso de un enfriador antes de la entrada del compresor 2. Se busca que el trabajo de compresión se disminuya con el enfriamiento de dicha corriente de flujo y, por ende, obtener una mayor potencia neta en el ciclo.

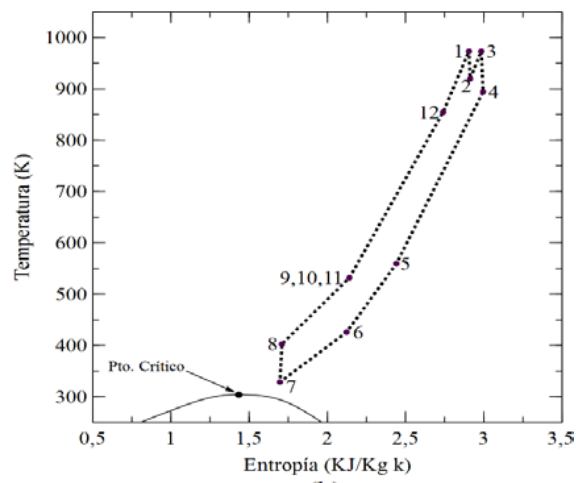

(b)

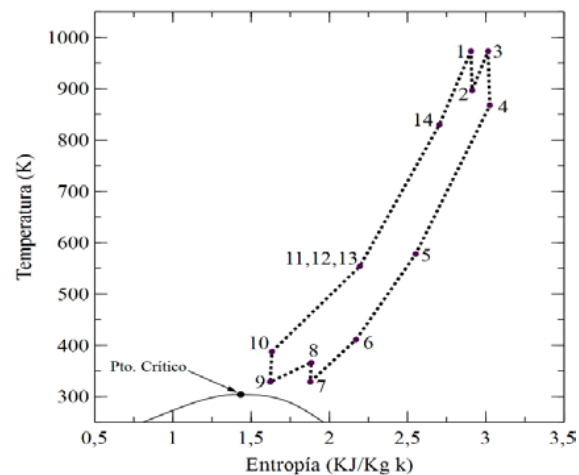

(d)

Fig. 3. Diagramas de temperatura-entropía (T-S) de las diferentes configuraciones de ciclo Brayton s- $\mathrm{CO}_{2}$ con recalentamiento a una temperatura de $700{ }^{\circ} \mathrm{C}$. (a) Conf. simple, (b) Conf. RC, (c) Conf. PC, (d) Conf. MC. Fuente: elaboración propia. 


\section{III.VALIDACIÓN Y MODELOS REALIZADOS}

Con el fin de garantizar que los resultados obtenidos en las simulaciones de las cuatro configuraciones propuestas de ciclo Brayton s- $\mathrm{CO}_{2}$ sean adecuados, se procedió a realizar la previa validación de los modelos realizados. Los diagramas temperatura entropía (T-S) para las configuraciones estudiadas (anteriormente mencionadas), se presentan en la Fig. 3.

\section{A. Modelo de energía}

Balances de masa y energía son llevados a cabo en cada uno de los intercambiadores de calor y otros componentes pertenecientes a las configuraciones propuestas. Debido a la rápida variación de las propiedades termo-físicas del fluido de trabajo cerca del punto crítico de operación [9] [13], se hace necesario discretizar el intercambiado de calor con el fin de corroborar si ocurren problemas con la temperatura de pinch point [20] [21] [22]. Para todas las configuraciones, los recuperadores son modelados asumiendo la efectividad de los intercambiadores de calor.

$$
\varepsilon_{\text {hot }, \text { stream }}=\frac{h_{4}-h_{6}}{h_{4}-h_{6 @ T c}}
$$

La entalpía , es la entalpía del estado 6 calculada en función de la mínima temperatura con la que la corriente caliente sale del recuperador de baja temperatura (LTR, por sus siglas en inglés); es así como se tiene que para el ciclo de recompresión es igual $\mathrm{a}$, mientras que para $\mathrm{PC}$ y MC es igual a [9]. El parámetro de la efectividad de los intercambiadores de calor es utilizado para todas las configuraciones que hagan uso del LTR, sin embargo, la efectividad del LTR es calculada.

Otro parámetro importante usado en las configuraciones que hacen uso del LTR es el split ratio (SR), el cual es la fracción del flujo que pasa a través de la línea de baja temperatura del LTR. El SR es determinado aplicando un balance de masa en el LTR [9]:

$$
S R=\frac{h_{5}-h_{6}}{h_{11}-h_{c}}
$$

Donde se tiene que es la entalpía de la linea fría que entra al LTR.

La eficiencia del ciclo según la primera ley de la termodinámica es calculada como:

$$
\eta_{I, \text { cycle }}=\frac{\dot{W}_{\text {net,turbina }}-\dot{W}_{\text {net,compresor }}}{\dot{Q}_{\text {calentador }}+\dot{Q}_{\text {recalentador }}}
$$

Los modelos desarrollados fueron escritos y ejecutados en el entorno de MatLab V2015a [23] con el complemento REFPROP 9.1 [24], usado para la obtención de las propiedades termodinámicas del fluido de trabajo. Con el fin de optimizar la eficiencia térmica de las configuraciones propuestas, las condiciones óptimas fueron obtenidas con el uso del código genético Particle Swarm Optimization (PSO) [25].

Las suposiciones tenidas en cuenta durante las simulaciones fueron [8] [9] [26]:

- Las caídas de presión en las tuberías y a través de los intercambiadores de calor son despreciadas;

- todos los componentes del ciclo están bien aislados;

- el ciclo trabaja bajo condiciones de estado estacionario;

- el dióxido de carbono siempre alcanza mínima y máxima temperatura y presión del ciclo.

La primera de estas consideraciones se hace debido a que examinar las caídas de presión implicaría ceñir la investigación al tipo de configuración interna de los equipos de intercambio de calor, lo cual se sesga del presente estudio, que es sobre el análisis del máximo potencial que puede ser entregado por ciclos Brayton que utilizan $\mathrm{CO}_{2}$ como fluido de trabajo operando en condiciones supercríticas

El modelo energético es validado con los resultados presentados por [9] y otros autores [8] [14]. Los parámetros usados para el proceso de validación son mostrados en la Tabla 1.

Tabla 1. Parámetros de entrada usados PARA VALIDAR EL MODELO PROPUESTO.

\begin{tabular}{|c|c|}
\hline Parámetro & Valor \\
\hline Eficiencia de la turbina & $93 \%^{\mathrm{a}}$ \\
\hline Eficiencia del compresor & $89 \%$ \\
\hline Efectividad del intercambiador & $95 \%$ \\
\hline Temperatura de entrada de la turbina & $500^{\circ} \mathrm{C}-800^{\circ} \mathrm{C}$ \\
\hline Presión de alta del ciclo & $25 \mathrm{MPa}$ \\
\hline Mínima temperatura de pinch point & $5^{\circ} \mathrm{C}$ \\
\hline
\end{tabular}

${ }^{a}$ Una eficiencia del 90\% es tomada en el ciclo de recompresión.

Fuente: tomado de [9].

En la Fig. 4 se presentan los resultados de las eficiencias térmicas de las diferentes configuraciones. Los resultados obtenidos del modelo propuesto concuerdan adecuadamente con los resultados de referencia, con errores menores al 3,8 |\% (error relativo máximo) para todas las configuraciones. Estas pequeñas desviaciones se presentan debido a la base de datos de las propiedades térmicas usadas, al modelo y el código de optimización implementado o a la capacidad de procesamiento de los equipos empleados para las simulaciones. Los resultados comparativos del proceso de validación se pueden apreciar en la Tabla 2. Por lo tanto, los modelos propuestos en la presente investigación son extendidos hasta un análisis exergético. 

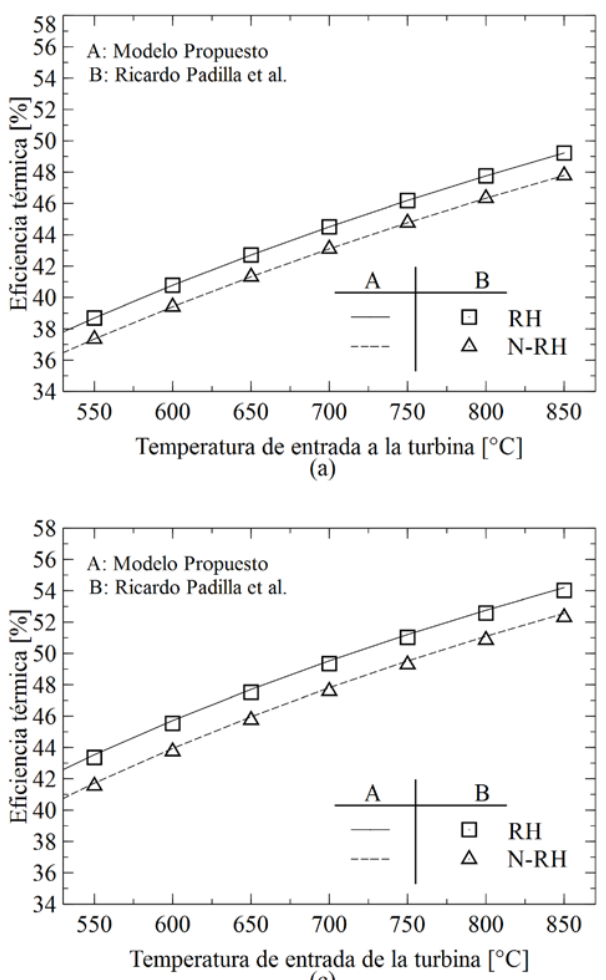

(c)

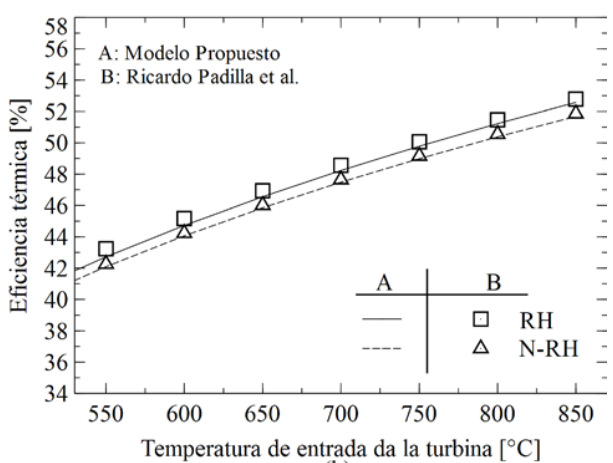

(b)

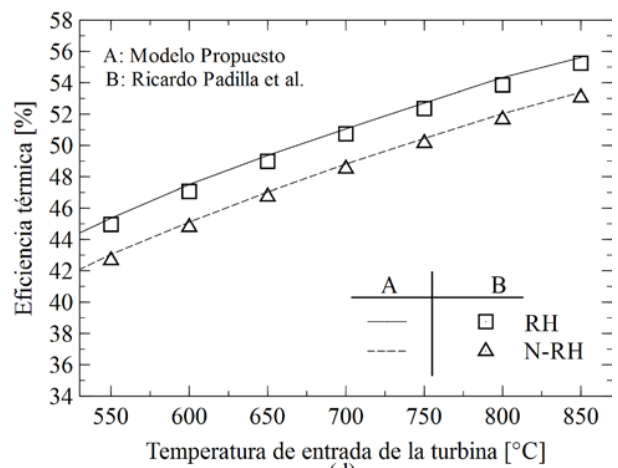

(d)

Fig. 4. Resultados gráficos del proceso de validación. Diferencias de los modelos desarrollados con respecto a los valores obtenidos de [9]. (a) Conf. simple, (b) Conf. RC, (c) Conf. PC, (d) Conf. MC.

Fuente: elaboración propia.

Tabla 2. Resultados numéricos del proceso de Validación. Diferencias de LOS MODELOS DESARROLLADOS CON RESPECTO A LOS VALORES OBTENIDOS POR [9].

\begin{tabular}{|c|c|c|c|c|c|}
\hline & & \multicolumn{2}{|c|}{ CON RECALENTADOR } & \multicolumn{2}{|c|}{ SIN RECALENTADOR } \\
\hline & ${ }^{\circ} \mathrm{C}$ & \multicolumn{2}{|c|}{ Eficiencia } & \multicolumn{2}{|c|}{ Eficiencia } \\
\hline CONFIGURACIÓN & Temperatura & Obtenidas $^{1}$ & $\mathrm{CSIRO}^{2}$ & Obtenidas $^{1}$ & $\mathrm{CSIRO}^{2}$ \\
\hline \multirow{4}{*}{ SIMPLE } & 650 & 0,4271 & 0,4271 & 0,4132 & 0,4132 \\
\hline & 700 & 0,4451 & 0,4451 & 0,4310 & 0,4310 \\
\hline & 750 & 0,4619 & 0,4619 & 0,4477 & 0,4477 \\
\hline & 800 & 0,4776 & 0,4776 & 0,4633 & 0,4633 \\
\hline \multirow{4}{*}{ RC } & 650 & 0,4655 & 0,4693 & 0,4557 & 0,4602 \\
\hline & 700 & 0,4823 & 0,4856 & 0,4726 & 0,4765 \\
\hline & 750 & 0,4978 & 0,5006 & 0,4882 & 0,4916 \\
\hline & 800 & 0,5122 & 0,5147 & 0,5027 & 0,5055 \\
\hline \multirow{4}{*}{$\mathbf{P C}$} & 650 & 0,4797 & 0,4752 & 0,4616 & 0,4575 \\
\hline & 700 & 0,4982 & 0,4935 & 0,4803 & 0,4759 \\
\hline & 750 & 0,5152 & 0,5103 & 0,4975 & 0,4929 \\
\hline & 800 & 0,5309 & 0,5258 & 0,5134 & 0,5086 \\
\hline \multirow{4}{*}{ MC } & 650 & 0,4966 & 0,4898 & 0,4728 & 0,4673 \\
\hline & 700 & 0,5145 & 0,5074 & 0,4909 & 0,4851 \\
\hline & 750 & 0,531 & 0,5236 & 0,5075 & 0,5014 \\
\hline & 800 & 0,5463 & 0,5386 & 0,5228 & 0,5166 \\
\hline
\end{tabular}

${ }^{1}$ Modelo de elaboración propia. ${ }^{2}$ Modelo de referencia de Padilla et al. [9]

Fuente: elaboración propia. 
En la Fig. 5 y Fig. 6 se observan las curvas de eficiencia térmica para todas las configuraciones del modelo desarrollado.

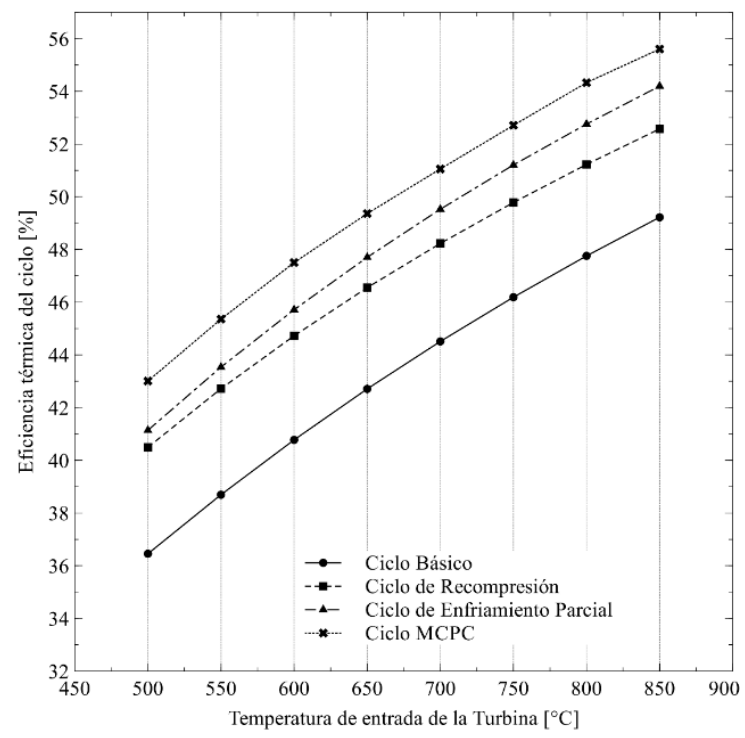

Fig. 5. Eficiencias térmicas de las diferentes configuraciones del ciclo Brayton s- $\mathrm{CO}_{2}$ con recalentamiento a una temperatura de $700^{\circ} \mathrm{C}$. Fuente: elaboración propia.

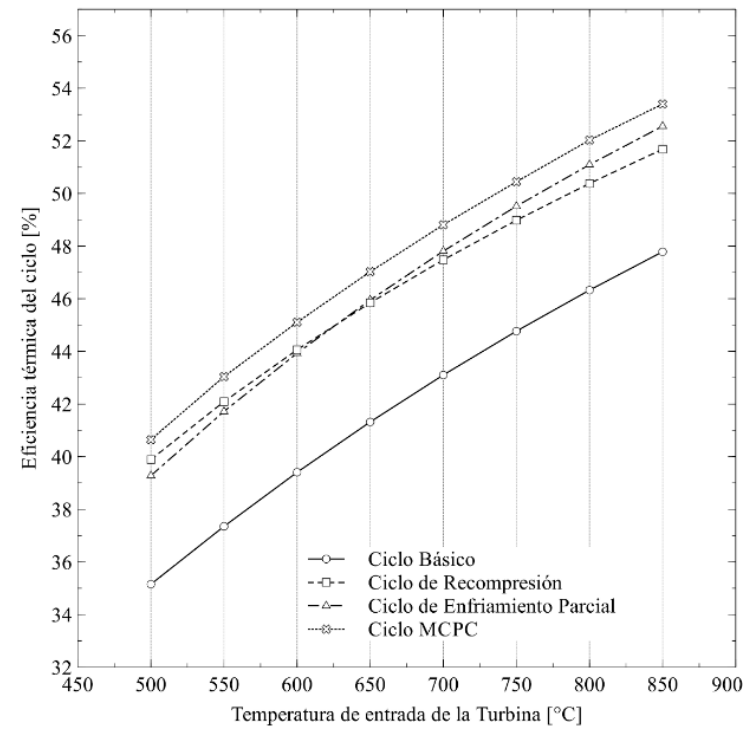

Fig. 6. Eficiencias térmicas de las diferentes configuraciones del ciclo Brayton s- $\mathrm{CO}_{2}$ sin recalentamiento a una temperatura de $700^{\circ} \mathrm{C}$. Fuente: elaboración propia.

\section{B. Modelo de exergía}

El balance de exergía para cada uno de los componentes que hacen parte del ciclo es calculado de la siguiente forma:

$$
\begin{aligned}
\frac{d E_{v . c .}}{d t}= & \sum_{j} \dot{E}_{q j}-\dot{W}_{c v}+\sum_{j} \dot{m}_{i} b_{i} \\
& -\sum_{o} \dot{m}_{o} b_{o}-\dot{E}_{d}-\dot{E}_{\text {loos }}
\end{aligned}
$$

Donde se tiene que:

$$
\begin{aligned}
& \dot{E}_{q}=\left(1-\frac{T_{0}}{T_{j}}\right) \dot{Q}_{j} \\
& b=h-h_{o}-T_{o}\left(s-s_{o}\right)+\frac{V^{2}}{2}+g z
\end{aligned}
$$

En esta investigación, los efectos térmicos producidos por la fuente térmica que provee la energía de entrada al ciclo no se tienen en cuenta en el análisis, ya que se maneja esta fuente térmica como una "caja negra" de la cual solo se extrae el calor requerido para el funcionamiento de las configuraciones propuestas, ya que el análisis se enfoca en el ciclo de generación de potencia, como se puede apreciar en la Fig. 1.

La eficiencia exergética del ciclo es calculada de dos formas distintas con la aplicación de las ecuaciones (7) y (8) con el fin de verificar los resultados obtenidos.

$$
\begin{aligned}
& \eta_{\text {exergy }}=\frac{\dot{W}_{\text {net, turbina }}-\dot{W}_{\text {net,compresor }}}{\dot{E}_{\text {input }}} \\
& \eta_{\text {exergy }}=1-\frac{\sum\left(\dot{E}_{\text {loss, comp }}+\dot{E}_{d, \text { comp }}\right)}{\dot{E}_{\text {input }}}
\end{aligned}
$$

En la Fig. 7 se presentan los resultados de las eficiencias exergéticas para todas las configuraciones estudiadas en presencia y ausencia de recalentamiento, notándose claramente un aumento de la eficiencia exergética cuando se hace uso del recalentador.

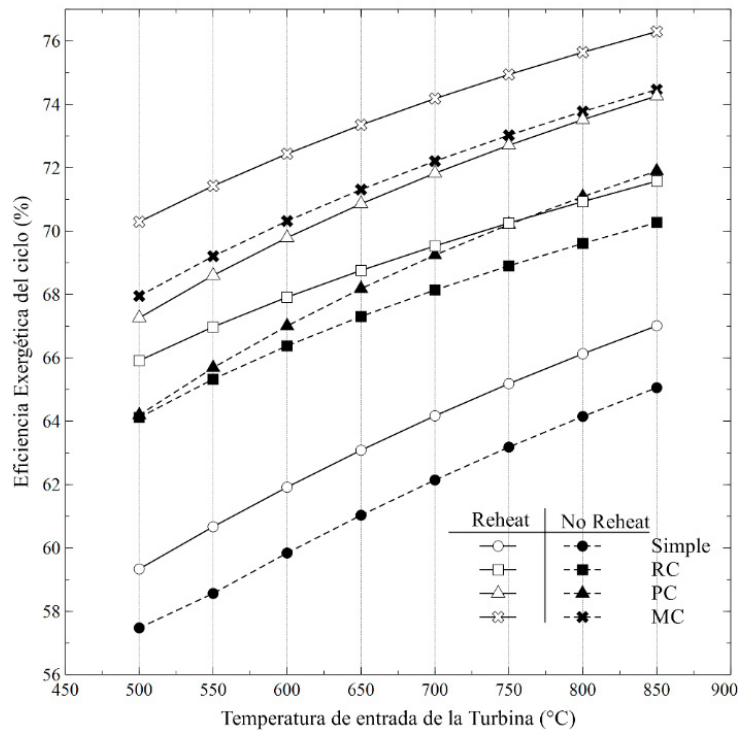

Fig. 7. Eficiencias exergéticas de las diferentes configuraciones de un ciclo Brayton $\mathrm{s}-\mathrm{CO}_{2}$ con recalentamiento y sin recalentamiento a una temperatura de $700^{\circ} \mathrm{C}$. Fuente: elaboración propia. 


\section{III.RESULTADOS Y ANÁLISIS}

Con el fin de comparar los modelos propuestos con la información disponible en la bibliografía, se realizó la previa validación de los resultados obtenidos con las simulaciones llevadas a cabo. El análisis de los datos arroja que los modelos realizados presentan un leve error máximo del 3,8\% (de error relativo) para todas las configuraciones con respecto a los datos de referencia [9].

De esta forma, tal como se aprecia en la Fig. 5, se tiene que la configuración que presenta la más alta eficiencia térmica es la de compresión principal con $43,1 \% @ T=500^{\circ} \mathrm{C}-56,0 \% @ T=850^{\circ} \mathrm{C}$ interenfriamiento parcial y recalentamiento (MC) con mientras que la configuración simple sin recalentamiento presenta la más baja eficiencia térmica de todas las configuraciones estudiadas con $35,1 \% @ T=500^{\circ} \mathrm{C}-47,7 \% @ T=850^{\circ} \mathrm{C}$

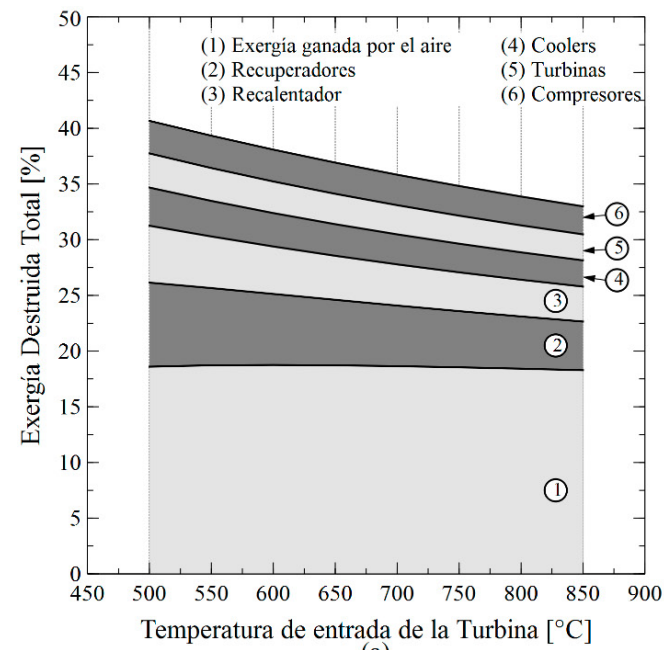

(a)

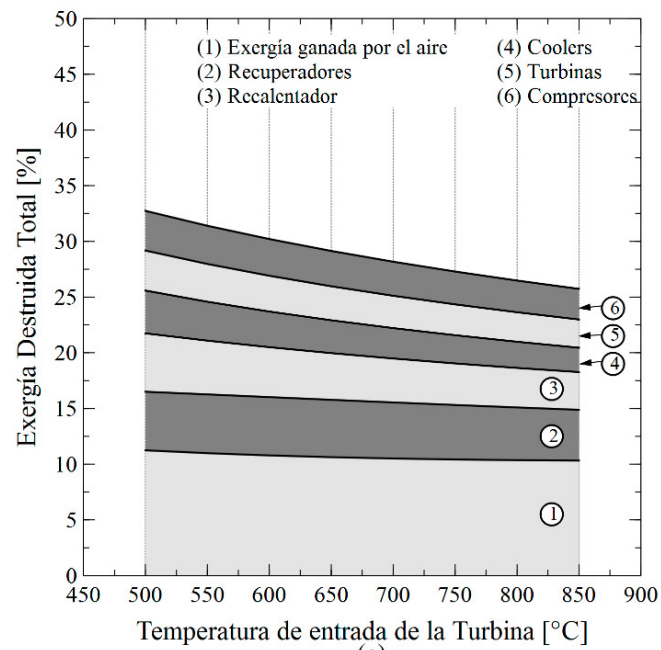

(c)
Estos resultados corroboran que la combinación de componentes como el interenfriador, el recompresor, el recuperador y el recalentador usados en un ciclo Brayton supercrítico puede lograr eficiencias térmicas mayores que una planta ultrasupercrítica (USC, Ultra-Supercritical en inglés), la cual puede alcanzar una eficiencia de alrededor del $47 \%$ operando entre $732-760^{\circ} \mathrm{C}$ y $35 \mathrm{MPa}$ [9] [27].

Por otra parte, un análisis en función de la segunda ley de la termodinámica en los ciclos de potencia usualmente ayuda a predecir el nivel de irreversibilidad de los componentes que hacen parte de las configuraciones del ciclo y, así, cuantificar la generación de entropía de los componentes, lo cual es de mucha importancia para minimizar las pérdidas totales de exergía y mejorar la eficiencia global del ciclo [16].

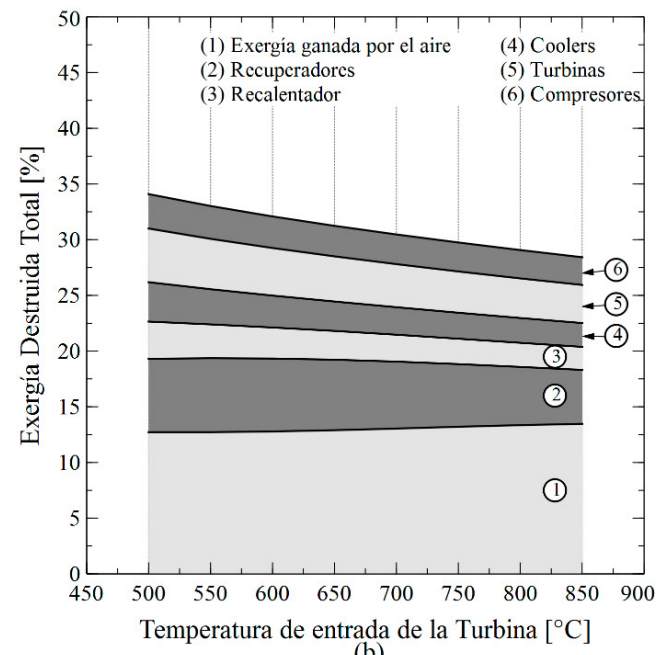

(b)

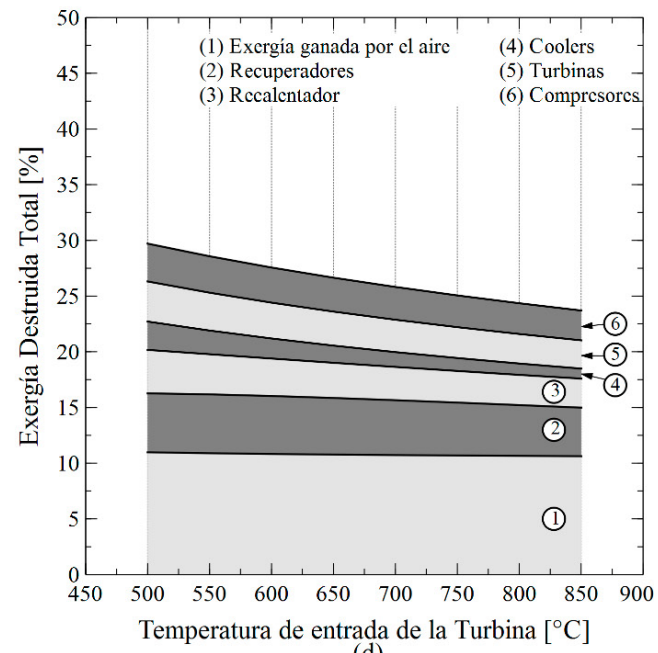

(d)

Fig. 8. Pérdidas totales de exergía por componentes para diferentes configuraciones del ciclo Brayton supercrítico con recalentamiento. (a) Conf. simple, (b) Conf. RC, (c) Conf. PC, (d) Conf. MC. Fuente: elaboración propia. 
Los resultados obtenidos en la presente investigación, demuestran que las pérdidas totales de exergía para ciclos con recalentamiento son consistentemente más bajas que aquellas configuraciones sin recalentamiento. La disminución promedio en puntos porcentuales para cada configuración con recalentamiento con respecto a la misma configuración sin recalentamiento es: configuración simple (simple) -3,87\%, configuración de recompresión (RC) $-1,87 \%$, enfriamiento parcial con recompresión (PC) -3,30\% y compresión principal con interenfriamiento parcial (MC) $-3,74 \%$.

Un análisis de las pérdidas totales de exergía por componente de las cuatro configuraciones del ciclo Brayton supercrítico, mediante el uso del recalentador y sin este, es presentado en la Fig. 8 y la Fig. 9, respectivamente. Se tiene que, para el caso de las configuraciones sin recalentador, las pérdidas de exergía son considerablemente mayores, sin embargo, se produce una disminución de éstas a medida que aumenta la temperatura. En el rango de temperaturas analizado, se observó una disminución de la exergía destruida del $14 \%$ para las configuraciones con recalentamiento, lo cual se acentúa a medida que la temperatura aumenta.

Por otra parte, a pesar de que las configuraciones que implementan el uso de recalentador poseen las menores pérdidas totales de exergía, estas presentan una disminución del $13 \%$ entre la temperatura más baja y la más alta de las analizadas para todas las configuraciones. Se puede apreciar, de igual forma, que, tanto para las configuraciones con recalentamiento como sin este, las mayores pérdidas exergéticas se presentan por la exergía ganada por el aire de enfriamiento, teniendo que para el caso de las configuraciones con recalentamiento están entre el $11 \%$ y $16 \%$ de las pérdidas totales; mientras que para las configuraciones sin recalentamiento, las pérdidas de exergía debido a la exergía ganada por el aire están en un rango de 10 y $15 \%$ de las pérdidas totales teniendo en cuenta todas las temperaturas analizadas.

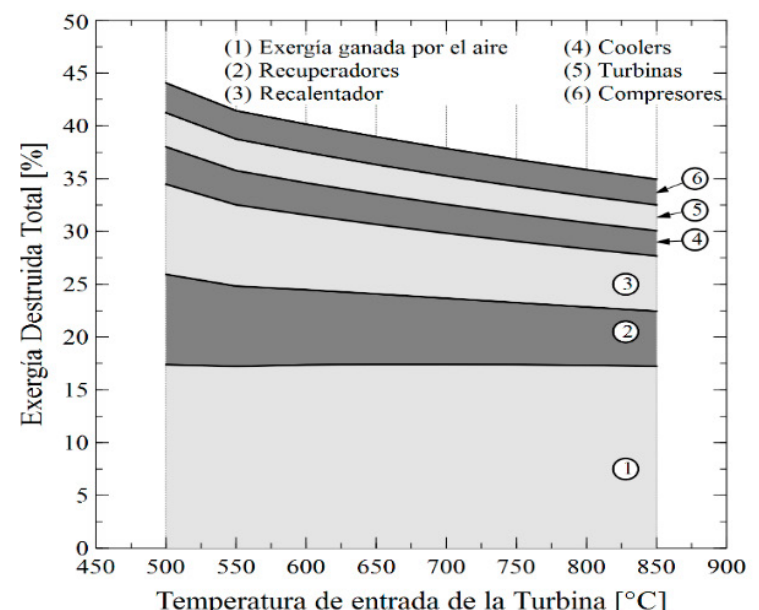

(a)

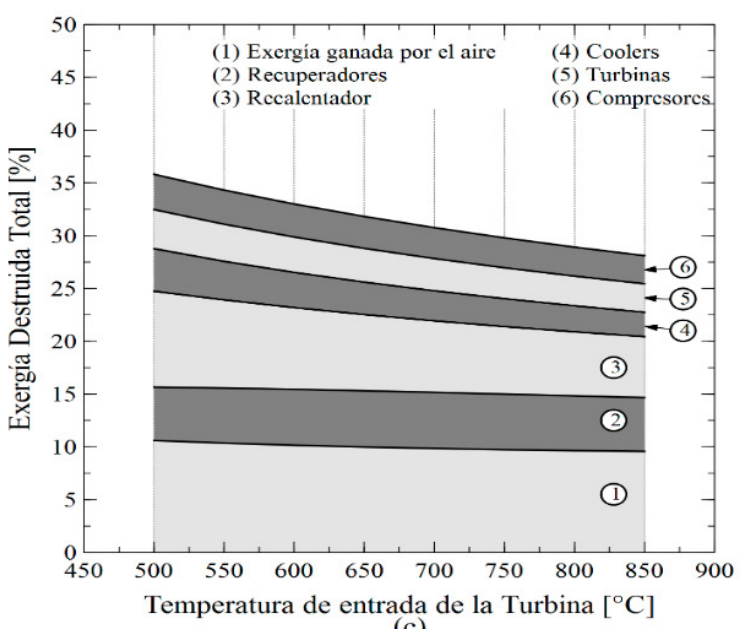

(c)

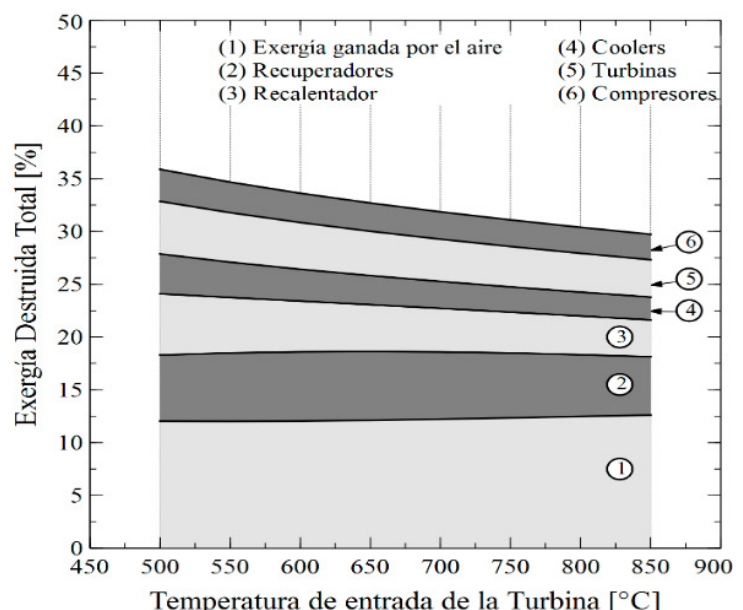

(b)

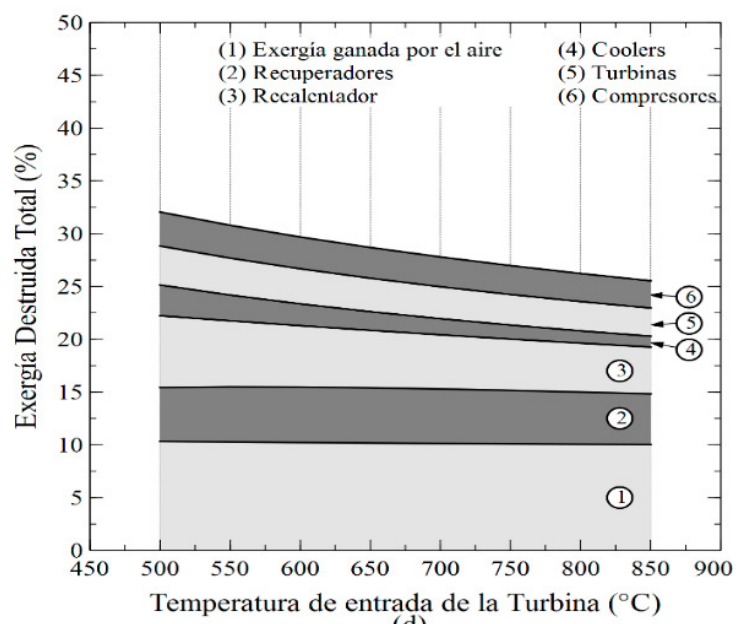

(d)

Fig. 9. Pérdidas totales de exergía por componentes para diferentes configuraciones del ciclo Brayton supercrítico sin recalentamiento. (a) Conf. simple, (b) Conf. RC, (c) Conf. PC, (d) Conf. MC. Fuente: elaboración propia. 
Por su parte, los componentes que menos aportan pérdida de exergía al ciclo son (en promedio) los compresores seguidos por las turbinas, los cuales oscilan entre un $5 \%$ y un $7 \%$. Sin embargo, cabe resaltar que los coolers o enfriadores que se encuentran ubicados antes de los compresores presentan un comportamiento interesante en el cual, a bajas temperaturas, las pérdidas exergéticas disminuyen notablemente más rápido que los demás componentes con el aumento de la temperatura, sin importar si se usa recalentador o no, situación que se hace más notable en la configuración MC.

\section{Conclusiones}

Un análisis térmico en función de la primera y segunda ley de la termodinámica de un ciclo Brayton supercrítico con dióxido de carbono $\left(\mathrm{s}-\mathrm{CO}_{2}\right)$ como fluido de trabajo fue llevado a cabo con el fin de realizar un posterior análisis del uso de los componentes implementados y así determinar el impacto de estos en cada configuración analizada, las cuales son: configuración simple, configuración de recompresión (RC), configuración de enfriamiento parcial (PC) y la configuración de compresión principal con interenfriamiento parcial (MC). El modelo térmico desarrollado demostró que se ajusta de manera adecuada al comportamiento descrito en la documentación bibliográfica previamente revisada.

Con base en los resultados obtenidos, las siguientes observaciones finales son propuestas:

- La configuración de compresión principal con interenfriamiento parcial y recalentamiento presenta las mejores eficiencias térmicas del ciclo dentro de los rangos de temperatura analizados $\left(43,1 \% @ T=500^{\circ} \mathrm{C}-56,0 \% @ T=850^{\circ} \mathrm{C}\right)$. Además, muestra el mejor comportamiento exergético respecto a las demás configuraciones.

- La configuración simple sin recalentamiento presenta las más bajas eficiencias térmicas (35,1\%@ $T=500^{\circ} \mathrm{C}-47,7 \%_{@ T=850^{\circ} \mathrm{C}}$ y exergéticas de las cuatro configuraciones estudiadas. Sin embargo, por su simplicidad y al ser mucho más compacto que las demás configuraciones, su uso sigue siendo muy atractivo.

- La adición del recalentador a las configuraciones del ciclo Brayton s- $\mathrm{CO}_{2}$ es una opción muy llamativa, ya que ayuda a mejorar considerablemente el rendimiento térmico del ciclo en al menos un 2,5\%, en el mejor de los casos.

- Los resultados teóricos obtenidos desde el punto de vista termodinámico (primera y segunda ley) muestran que el ciclo Brayton supercrítico es una buena opción para la generación de potencia trabajando en un rango de temperaturas de $550^{\circ} \mathrm{C}$ a $850^{\circ} \mathrm{C}$. Sin embargo, debido a que en la presente investigación fueron omitidos los efectos exergéticos causados por la fuente térmica (ya que esta fue tomada como una caja negra, como fue expuesto anteriormente), se recomienda para futuras investigaciones llevar a cabo el análisis incluyendo tales efectos exergéticos y evaluar nuevamente los resultados con el fin de determinar si dicha fuente impacta radicalmente en los nuevos datos. Vale resaltar que dependiendo de qué tipo de tecnología sea usada como fuente térmica, así mismo se espera que los resultados exergéticos tengan una considerable variación, ya que de la capacidad y eficiencia de captación y transferencia de calor de dicha fuente dependerán los resultados exergéticos.

- La implementación de enfriadores e interenfriadores en las configuraciones del ciclo mejora considerablemente el rendimiento de las configuraciones estudiadas, disminuyendo el consumo de potencia requerido por los compresores $\mathrm{y}$, por ende, permitiendo un mejor aprovechamiento de la potencia generada por las turbinas.

- El uso del recalentador favorece a la disminución de las pérdidas totales de exergía del ciclo respecto a las configuraciones en las que no se lleva a cabo su uso, presentándose en promedio una disminución del 3,87 \% para la configuración simple y del $3,74 \%$ en la configuración MC, siendo estas configuraciones las que presentan la disminución más notable.

- Las pérdidas totales de exergía son mayores en las configuraciones que implementan el uso de recalentador que en aquellas donde no lo usan. Se aprecia que, con el uso del recalentador, las pérdidas de exergía disminuyen en al menos un $3 \%$ a medida que aumenta la temperatura para todas las configuraciones.

\section{Financiación}

Artículo de investigación derivado del proyecto de grado titulado "Análisis exergético de un ciclo Brayton supercrítico con dióxido de carbono como fluido de trabajo" desarrollado por Moisés Herrera y Edgardo Castro para optar al título de ingenieros mecánicos por parte de la Universidad del Atlántico. El proyecto fue dirigido por el Dr. Ing. Jorge Duarte Forero y codirigido por el Ing. Armando Fontalvo. A lo largo del proyecto se recibió apoyo por parte de la Southern Cross University en Australia a través del Dr. Ricardo Vásquez. Fecha de inicio: enero de 2017. Fecha de finalización: marzo de 2018.

\section{REFERENCIAS}

[1] F. Barrozo, G. Valencia y Y. Cárdenas, "An economic evaluation of renewable and conventional electricity generation systems in shopping center using HOMER Pro", Contemporary Engineering Sciences, vol. 10, no. 26, 1287$1295,2017$.

[2] G. Amador, J. Duarte, J. García, A. Rincón, A. Fontalvo, A. Bula y R. Padilla, "Maximun power from fluid flow by applying the first and second laws of thermodynamics", Journal of Energy Resources Technology, ASME, vol. 139, no. 3, 2016. http://dx.doi.org/10.1115/1.4035021 
[3] H. Chen, "The conversion of low-grade heat into power using supercritical Rankine cycles," tesis de maestría, University of South Florida, 2010.

[4] A. I. Kalina, "Combined cycle and waste heat recovery power systems based on a novel thermodynamic energy cycle utilizing low-temperature heat for power generation", American Society of Mechanical Engineers, Turbo Expo: Power for Land, Sea, and Air, at the Joint Power Generation Conference: GT papers, 1983. https://doi. org/10.1115/83-JPGC-GT-3

[5] Y. D. Goswami, "Solar thermal power technology: present status and ideas for the future", Energy sources, vol. 20 , no. 2 , pp. 137-145, 1998. https://doi. org/10.1080/00908319808970052

[6] A. Fontalvo, H. Pinzón, J. Duarte, A. Bula, A. González, "Exergy analysis of a combined power and cooling cycle", Applied Thermal Engineering, vol. 60, no. 1, pp. 164-171, 2013. https://doi.org/10.1016/j. applthermaleng.2013.06.034

[7] E. D. Rogdakis y K. A. Antonopoulos, "A high efficiency NH3/H2O absorption power cycle", Heat Recovery Systems and CHP, vol. 11, no. 4, pp. 263-275, 1991. https:// doi.org/10.1016/0890-4332(91)90072-C

[8] C. S. Turchi, Z. Ma, T. W. Neises y M. J. Wagner, "Thermodynamic study of advanced supercritical carbon dioxide power cycles for concentrating solar power systems", Journal of Solar Energy Engineering, vol. 135, no. 4, 2013. https://doi.org/10.1115/1.4024030

[9] R. Padilla, Y. Chen, R. Benito y W. Stein, "Exergetic analysis of supercritical $\mathrm{CO}_{2}$ Brayton cycles integrated with solar central receivers", Applied Energy, vol. 148, pp. 348. 365, 2015. https://doi.org/10.1016/j.apenergy.2015.03.090

[10] R. Padilla, A. Ramos, G. Demirkaya, S. Besarati, D. Goswami, M. Rahman y E. Stefenakos, "Performance analysis of a Rankine cycle integrated with the Goswami combined power and cooling cycle", Journal of Energy Resources Technology, vol. 143, no. 3, 2012.

[11] Tzu-Chen Hung, "Waste heat recovery of organic Rankine cycle using dry fluids", Energy Conversion and Management, vol. 42 , no. 5, pp. 539-553, 2001. https://doi. org/10.1016/S0196-8904(00)00081-9

[12] Y. Chen, P. Lundqvist, A. Johansson y P. Platell, "A comparative study of the carbon dioxide transcritical power cycle compared with an organic Rankine cycle with R123 as working fluid in waste heat recovery", Applied Thermal Engineering, vol. 26, no. 17, pp. 2142-2147, 2006. https:// doi.org/10.1016/j.applthermaleng.2006.04.009

[13] V. Dostal, M. J. Driscoll y P. Hejzlar, "A supercritical carbon dioxide cycle for next generation nuclear reactors", Dept. of Nuclear Engineering, Massachusetts Institute of Technology, 2004.

[14] M. D. V. Kulhánek, "Thermodynamic analysis and comparison of supercritical carbon dioxide cycles", de Proceedings of Supercritical $\mathrm{CO}_{2}$ Power Cycle Symposium, 2011.
[15] A. Moisseytsev y J. J. Sienicki, "Investigation of alternative layouts for the supercritical carbon dioxide Brayton cycle for a sodium-cooled fast reactor", Nuclear Engineering and Design, vol. 239, no. 7, pp. 1362-1371, 2009. https://doi.org/10.1016/j.nucengdes.2009.03.017

[16] J. Sarkar, "Second law analysis of supercritical $\mathrm{CO}_{2}$ recompression Brayton cycle", Energy, vol. 34, no. 9, pp. 1172-1178, 2009. https://doi.org/10.1016/j. energy.2009.04.030

[17] A. Moisseytsev y J. Sienicki, "Performance improvement options for the supercritical carbon dioxide Brayton cycle," [Tech. rep. ANL-GenIV-103] Argonne National Laboratory (ANL), Chicago, 2007. http://www.ipd.anl.gov/ anlpubs/2008/07/61951.pdf

[18] E. G. Feher, "The supercritical thermodynamic power cycle", Energy conversion, vol. 8, no. 2, pp. 85-90, 1968. https://doi.org/10.1016/0013-7480(68)90105-8

[19] M. J. Moran y H. N. Shapiro, Fundamentals of engineering thermodynamics, 5th ed., Hoboken, N. J.: John Wiley and Sons, 2004.

[20] S. P. Kao, J. Gibbs y P. Hejzlar, "Dynamic simulation and control of a supercritical $\mathrm{CO}_{2}$ power conversion system for small light water reactor applications", Proceedings of supercritical $\mathrm{CO}_{2}$ power cycle symposium, 2009.

[21] H. T. Hoang, M. R. Corcoran y J. W. Wuthrich, "Thermodynamic study of a supercritical $\mathrm{CO}_{2}$ Brayton cycle concept", Proceedings of supercritical $\mathrm{CO}_{2}$ power cycle symposium, 2009.

[22] W. Seidel, "Model development and annual simulation of the supercritical carbon dioxide Brayton cycle for concentrating solar power applications", tesis doctoral, University of Wisconsin-Madison, 2011.

[23] Mathworks, Matlab. Computer Program. Massachusetts: MATLAB, 2014

[24] E. W. Lemmon, M. L. Huber y M. O. McLinden, "NIST standard reference database 23: reference fluid thermodynamic and transport properties-REFPROP", [Tech. rep.], version 9.1. Standard Reference Data Program, National Institute of Standards and Technology, Gaithersburg, 2013.

[25] S. Mishra, "Some new test functions for global optimization and performance of repulsive particle swarm method", 2006. Disponible en: SSRN 926132.

[26] S. M. Besarati y D. Y. Goswami, "Analysis of advanced supercritical carbon dioxide power cycles with a bottoming cycle for concentrating solar power applications", $J$. Sol. Energy. Eng, vol. 136, no. 1, 010904-1-7, 2014.

[27] R. Viswanathan, K. Coleman y U. Rao, "Materials for ultra-supercritical coal-fired power plant boilers", International Journal of Pressure Vessels and Piping, vol. 83, no. 11, pp. 778-783, 2006. https://doi.org/10.1016/j. ijpvp.2006.08.006 
Moisés Herrera Palomino es tesista aspirante al título de ingeniero mecánico de la Universidad del Atlántico y estudiante de Ingeniería Industrial del mismo centro universitario. Sus intereses son el modelado de sistemas termodinámicos y el modelado a través de paquetes de Ingeniería Asistida por Computador (Computer-Aided Engineering, CAE). https:// orcid.org/0000-0003-3236-5066

Edgardo Castro Pacheco es tesista aspirante al título de ingeniero mecánico de la Universidad del Atlántico. Actualmente es estudiante de séptimo semestre de Física de esta misma universidad. Sus intereses son el modelado de sistemas termodinámicos y el modelado a través de paquetes de Ingeniería Asistida por Computador (Computer-Aided Engineering, CAE). https://orcid.org/0000-0003-4765-0556

Jorge Duarte Forero es ingeniero mecánico y doctor en Ingeniería Mecánica. Sus intereses son el modelado de sistemas termodinámicos y el modelado a través de paquetes de Ingeniería Asistida por Computador (Computer-Aided Engineering, CAE). Es investigador asociado de Colciencias y profesor asociado en la Universidad del Atlántico. https://orcid.org/0000-0001-7345-9590
Armando Fontalvo Lascano es ingeniero mecánico y magíster en Ingeniería Mecánica. Sus intereses son el modelado de sistemas termodinámicos y el modelado a través de paquetes de Ingeniería Asistida por Computador (Computer-Aided Engineering, CAE). Es investigador junior de Colciencias y profesor asistente III en la Universidad de la Costa. https:// orcid.org/0000-0002-3445-1649

Ricardo Vásquez Padilla es profesor titular y coordinador de curso de la Licenciatura en Ingeniería Mecánica en la Escuela de Medio Ambiente, Ciencia e Ingeniería de Southern Cross University en Australia. Sus intereses de investigación incluyen el modelado y la experimentación de bloques avanzados de energía que operan con diferentes fuentes de calor, sistemas solares térmicos concentrados, sistemas híbridos de energía renovable y aplicaciones de energía renovable para calefacción, refrigeración y desalinización. http://orcid.org/0000-0002-4120-9711 